Journal-Ref: Astronomy Letters, 2010, Vol. 36, No. 11, pp. 808-815

\title{
Modulation of Circumstellar Extinction in a Young Binary System with a Low-Mass Companion in a Noncoplanar Orbit
}

\author{
V. P. Grinin ${ }^{1,2}$, T. V. Demidova ${ }^{1,2}$, N. Ya. Sotnikova ${ }^{1}$ \\ 1 - Sobolev Astronomical Institute, St. Petersburg State University, Universitetskii pr. 28, St. Petersburg, \\ 198504 Russia, \\ 2 - Pulkovo Astronomical Observatory, Russian Academy of Sciences, Pulkovskoe shosse 65, St. \\ Petersburg, 196140 Russia,
}

Received April 10, 2010

\begin{abstract}
The cyclic activity model of a young star with the low-mass secondary component $\left(q=M_{2} / M_{1} \leq\right.$ 0.1 ) accreting a matter from circumbinary disk is considered. It is assumed that the orbit is circular and the disk and orbital planes are non-coplanar. Sets of hydrodynamics models of such a system have been calculated by the SPH method and then the variations of the circumstellar extinction and phase light curves were determined. The calculations showed that depending on the model parameters and orientation of the system in regards to an observer the different in shape and amplitude light curves can be observed. An important property of the considered models is also the dependence of the mass accretion rate onto the components on the phase of the orbital period. The results of the calculation can be used for analysis of the cyclic activity of UX Ori stars and young stars with the long-lasting eclipses.

Key words: young binary systems, extinction modulation, activity cycles of UX Ori stars.
\end{abstract}




\section{INTRODUCTION}

In the previous papers of this series (Sotnikova and Grinin 2007; Demidova et al. 2010a, $2010 \mathrm{~b}$ ), we studied the behavior of the circumstellar extinction and brightness in young binary systems accreting matter from the remnants of a protostellar cloud. This problem was initiated by the observations of large-scale activity cycles in UX Ori stars (Grinin et al. 1998) and by the observations of anomalously long eclipses in some young stars (Cohen et al. 2003; Barsunova et al. 2005; Nordhagen et al. 2006; Grinin et al. 2008), which cannot be explained in terms of classical models of eclipsing binary systems. Sotnikova and Grinin (2007) and Demidova et al. (2010a, 2010b) showed that the streams of matter and density waves produced in young binary systems by the orbital motion of their companions can cause (at low inclinations of the orbital plane to the line of sight) various (in shape and duration) periodic fadings of the primary component. One of the main conditions in the computations was coplanarity of the orbital plane and the circumbinary (CB) disk.

In this paper, we consider the more general case where the orbit of the companion and the $\mathrm{CB}$ disk are noncoplanar. The prototype of such a binary is the star $\beta$ Pictoris surrounded by an extended circumstellar disk (Smith and Terrile 1984) seen almost edge-on. Detailed studies of the disk image (Burrows et al. 1995) showed that its inner region was inclined by several degrees relative to the periphery. This peculiarity is considered as circumstantial evidence for the existence of a planet in the central part of the disk whose orbit is inclined with respect to the outer part of the disk (Burrows et al. 1995; Mouillet et al. 1997; Larwood and Papaloizou 1997). The fact that the disk of $\beta$ Pictoris is the first and so far the only example of a comparatively young $\left(\approx 10^{7} \mathrm{yr}\right)$ circumstellar disks tudied very thoroughly and that evidence for the existence of a companion in a noncoplanar orbit was found in this disk suggests that such situations are encountered in nature not so rarely. This circumstance served for us as an incentive to perform computations whose results are presented below.

\section{MODEL PARAMETERS AND THE COMPUTATIONAL METHOD}

We consider a model of a binary system with a low-mass companion moving in a circular orbit of radius $a$ inclined with respect to the CB-disk plane by angle $\theta$. In our computations, we took $\theta=10^{\circ}$. The other parameters of the problem are: the orbital phase-averaged accretion rate onto both components of the binary $\left(\dot{M}_{a}\right)$, the orbital inclination to the line of sight $(i)$, and the angle between the projection of the line of sight onto the orbital plane and the line of nodes $(\phi)$. The mass of the central star $M_{*}$ was taken to be $2 M_{\odot}$, a value typical of many UXOri stars (Rostopchina 1999). The orbital period is $P=5 \mathrm{yr}$.

\subsection{The Computational Method}

As in Artymowicz and Lubow (1996), we computed the hydrodynamic model in the isothermal approximation. This approach is justified, because, as our computations show, not the entire CB disk but only its comparatively small region near the inner boundary makes a major contribution to the circumstellar extinction variations in our models.

The hydrodynamic models of the binary under consideration were computed by the SPH (Smoothed Particle Hydrodynamics) method described in detail by Sotnikova (1996). Let us briefly recall the main features of the computations. In our implementation of the SPH method, the smoothing length was assumed to be constant and was specified in fractions of 
the orbital radius: $h=0.1 a$. At each point, this provided at least 30 neighboring points, over which the hydrodynamic quantities were averaged. The parameter $c$, which characterizes the viscosity and is the dimensionless speed of sound expressed in units of the companion's Keplerian velocity, was taken to be 0.05 (a "warm" disk). (Note that for the values of $M_{*}$ and $P$ adopted above and at a stellar temperature of $10^{4} \mathrm{~K}$ typical of UX Ori stars, this speed of sound corresponds to a gas temperature about $100 K$.) The number of test particles used in our SPH simulations was $6 \cdot 10^{4}$. As a rule, the computations were performed for several hundred periods. For each model, we computed the column density of test particles toward the primary component of the binary as a function of time expressed in units of the orbital period. The cross-sectional area of the column $s$ was taken to be $2 h \times 2 h$. Our computations showed this value of $s$ to be optimal for the solution of our problem.

The technique for calculating the phase dependence of the dust column density was described in detail by Demidova et al. (2010a). Let us briefly recall its main points. Our quantitative analysis of the computational results begins with the removal of the trend in the particle column density variations attributable to the decrease in their number in the binary through accretion onto its components. The initial binary relaxation stage (with a duration of several tens of revolutions), during which its matter distribution comes to a stable state, is excluded from consideration. The trend is modeled by a fifth-degree polynomial. Our computations showed that this provided a satisfactory removal of the trend for all of the models under consideration. Subsequently, we passed from the test particle column density $n(t)$ to the column density of real dust grains $n_{d}(t)$ (for details, see Demidova et al. 2010a). To reduce the influence of random fluctuations when the phase dependences of $n$ are computed, the current values of $n(t)$ are folded with the orbital period for a time interval of 50 binary revolutions.

As in our previous papers, we assumed the primary component to be the main source of optical radiation. To determine the optical depth $\tau$ between the star and the observer attributable to circumstellar dust, it is necessary to find the column density of test particles as a function of time and to determine the "mass" of one such particle. For this purpose, we determined the number of test particles accreting onto the binary components at each instant of time (for more detail, see Sotnikova and Grinin 2007). These values were averaged over the orbital phases and their sum was compared with the mean accretion rate onto both components adopted in the model, $\dot{M}_{a}$. The "mass" of a single test particle $m_{d}$ was determined from the formula $m_{d}=P \dot{M}_{a} / N_{a}$, where $P$ is the orbital period and $N_{a}$ is the total number of test particles accreting onto both components in one binary revolution. In our computations below, we took $\dot{M}_{a}$ to be $10^{-9}$ and $10^{-10} M_{\odot}$. As our computations showed, these values of the accretion rate are sufficient to produce a high-amplitude brightness modulation in the primary component.

Having determined the mass of a single test particle, we obtain the matter column density in $\mathrm{g} / \mathrm{cm}^{2}$. To calculate the optical depth of the dust on the line of sight, we should specify the opacity $\kappa$ per gram of matter. As in our previous papers, below we adopted an average (for the interstellar medium) dust-to-gas ratio of $1: 100$ and $\kappa=250 \mathrm{~cm}^{2} / \mathrm{g}$ typical of circumstellar extinction in Johnson's B photometric band (Natta and Whitney 2000).

The intensity of the radiation from young stars is known to consist of two parts: the intensity of the direct stellar radiation $I_{*}$ attenuated by a factor of $e^{-\tau}$ and the intensity of the radiation scattered by circumstellar dust $I_{s c}: I_{o b s}=I_{*} e^{-\tau}+I_{s c}$. The contribution of the scattered light to the total radiation from young stars typically does not exceed a few percent. Therefore, below, when analyzing the pattern of variability in the primary component, we took the intensity of the scattered radiation to be zero. The light variations of the primary component are expressed in magnitudes: $\Delta m=-2.5 \cdot \log I_{o b s}\left(I_{*}\right.$ is taken as unity). Hence 
it follows that $\Delta m \sim \tau$, and since $\tau$ is proportional to $\dot{M}_{a}, \Delta m \sim \dot{M}_{a}$. This relation allows the light curves computed for one value of $\dot{M}_{a}$ to be easily recalculated for other values of this parameter.

There is another useful relation that allows the light curves computed for a period of $5 \mathrm{yr}$ to be recalculated to a different value of $P$. It can be derived from the following considerations: according to the above formula for the test particle mass, $m_{d}$ is proportional to the orbital period $P$. On the other hand, when calculating $\tau$, we divide the number of particles in the column $n(t)$ by its cross-sectional area $s$. The latter is proportional to $a^{2} \sim P^{4 / 3}$. Taking this into account, we obtain $\Delta m \sim \tau \sim P^{-1 / 3}$.

\section{RESULTS}

\subsection{Model Distributions of Particles in a Binary}

Figures 1 and 2 show the distributions of test particles computed by the SPH method for two models with different component mass ratios: $q=0.1$ (model 1 ) and $q=0.01$ (model 2 ). The coordinate system is chosen in such a way that the XY plane coincides with the CB disk plane, the coordinate origin coincides with the binary's center of mass, and the Y axis coincides with the line of nodes. We see that in model 1 , the inner region of the CB disk is strongly deformed and inclined with respect of its periphery due to the gravitational perturbations produced by the companion's orbital motion. We see from the binary's sections in the YZ and XZ planes presented in Fig. 1 that the inner disk region is inclined not only with respect to the $\mathrm{Y}$ axis but also in the $\mathrm{YZ}$ section (i.e., with respect to the $\mathrm{X}$ axis). In other words, the inclination of the inner $\mathrm{CB}$ disk region does not coincide closely with the orbital inclination. This pattern of deformation of the CB disks tems from the fact that in the models with an inclined orbit, the angular momentum vector for the companion's orbital motion has two components, one of which is orthogonal to the disk plane and the other coincides with it and is directed (in the adopted coordinate system) along the X axis. The latter component produces the CB disk inclination in the YZ section. This pattern of deformation of the inner disk in the models with an inclined orbit was first described in the paper by Mouillet et al. (1997) cited above, where the particle dynamics was computed in the ballistic approximation. Our computations showed that this peculiarity is also retained in the hydrodynamic models computed by taking into account the gas pressure and viscosity.

The mass of the companion in model 2 is a factor of 10 lower than that in model 1 . Therefore, the CB disk deformation is much weaker. However, in contrast to model 1, the region within the orbit is filled with matter to a greater extent and we see from Fig. 2 that this matter has the shape of a disk, whose plane coincides neither with the orbital plane nor with the CB disk plane. This peculiarity in the distribution of matter within the companion's orbit is attributable to the same effect as the CB disk deformation in model 1 described above. Since the accretion disks of the binary components are formed from this matter, they will also be inclined with respect of the orbital plane, in which the primary and secondary components revolve around the binary's center of mass.

\subsection{Accretional Activity of the Binary Components}

Figure 3 shows the phase dependences of the accretion rate onto the binary components computed for models 1 and 2. Just as in the models with coplanar orbits (Artymowicz and Lubow 1996; Bate and Bonnell 1997; Demidova 2009), the low-mass component is the main accretor (because its orbit is close to the CB disk). However, in contrast to these models, 
the accretion rate onto the components in models 1 and 2 depends on the orbital phase even for a circular orbit. In this case, the pattern of modulation of the accretion rate depends on the mass of the companion. The accretion rate onto the low-mass component in model $2(q=0.01)$ has two maxima. They correspond to times that are close to the times of the companion's passage through the line of nodes (but that do not coincide with them). The picture is completely different in model 1 with a more massive companion: the accretion rate has only one maximum instead of two. In this case, the accretion rates onto the primary and secondary components change in antiphase.

\subsection{Model Light Curves}

Figures 4-7 show the light curves of the binary's primary component computed by the method described above. The computations were performed for several orbital inclinations to the line of sight and four angles specifying the position of the line of nodes relative to the observer: $\phi=0^{\circ}, 90^{\circ}, 180^{\circ}, 270^{\circ}$. (In the frame of reference adopted here, the angle $\phi=0$ corresponds to the case where the line of sight is orthogonal to the line of nodes and the observer in Fig. 1 is located to the right; the angles are measured in the direction of rotation of the binary). As in our previous papers, the range of orbital inclinations relative to the line of sight considered (from 0,15 , and $18^{\circ}$ ) is constrained by the finite number of test particles used in our computations and by the necessity of avoiding great statistical fluctuations in the particle column density at higher inclinations i. As was said above, to suppress the fluctuations, the current values of $n(t)$ for the chosen phase intervals were summed over 50 revolutions and were then averaged and smoothed.

We see from Figs. 4-6 that the light curves depend significantly not only on the orbital inclination to the line of sight but also on the position of the line of nodes. The contribution to the extinction from the CB-disk matter at different distances from its edge and from the stream propagating from the CB disk toward the secondary component (it is clearly seen in Figs. 1 and 2) changes with these parameters. As a result, light curves differing significantly in both amplitude and shape are obtained for different binary orientations. The strong influence of the orientation of the apsidal line relative to the observer on the light curves is caused by an azimuthal asymmetry of the inner CB disk gap. It is the CB-disk matter along the line of sight that causes a prolonged eclipse of the central star. The secondary, more compact minimum is produced by the absorption of radiation in the stream. The combined action of these two effects gives rise to two-component light curves (Figs. 4 and 5).

Situations similar to that described above are also possible in models with coplanar orbits (see Demidova et al. 2010a, 2010b). However, as our comparative analysis showed, other things being equal, the brightness modulation depth in models with noncoplanar CB disk and orbit is appreciably larger than that in coplanar binary systems. As a result, a noticeable (in amplitude) brightness modulation in a young star can be produced even by the motion of a companion with a mass corresponding to the mass of a giant planet in a noncoplanar orbit around it (Fig. 7).

\section{DISCUSSION AND CONCLUSIONS}

The above computations supplement the results obtained in our previous papers. They show what types of brightness oscillations may be expected in young binary systems with different orbital parameters and different companion masses. In all these models, the brightness variations are caused by periodic extinction variations and, therefore, depend strongly on the 
orbital inclination and orientation with respect to the observer. At low inclinations, a periodic brightness modulation can be observed even at very low accretion rates, $10^{-10} M_{\odot} / \mathrm{yr}$, typical of the final evolutionary stages of circumstellar disks.

In this case, a low-mass companion like a brown dwarf or even a giant planet can be the source of the perturbations causing the extinction variations. In our previous papers, we have already pointed out a great similarity of the model light curves with the activity cycles observed in UX Ori stars. These cycles have a wide variety of shapes. Simple cycles that can be satisfactorily described by a single sine wave are commonly observed (see, e.g., Artemenko et al. 2010). In some cases, the cycles are more complex in shape. For example, the photometric cycle of BF Ori about $11 \mathrm{yr}$ in duration has a two component structure (Grinin et al. 1998)1 that resembles the model light curves with two successive minima (Figs. 4-7). The activity cycle of CO Ori, which, according to Rostopchina et al. (2007), has been observed in this star for several decades, also has a similar shape. From the viewpoint of the models considered above, the two types of photometric cycles (simple and two-component) do not differ fundamentally. They can be obtained in terms of the same model with different orientations of the orbit relative to the observer. This peculiarity of the model we considered is its important advantage over other mechanisms of cyclic activity in UX Ori stars discussed in the literature.

\section{ACKNOWLEDGMENTS}

This work was supported by the "Origin and Evolution of Stars and Galaxies" Program of the Presidium of the Russian Academy of Sciences and the Program for Support of Leading Scientific Schools (NSh-3645.2010.2).

\footnotetext{
${ }^{1}$ The reality of this cycle is confirmed by the analysis of the photometric observations performed with the ASAS telescope and published in the catalog by Poimanski (2002).
} 


\section{REFERENCES}

1. S. A. Artemenko, K. N. Grankin, and P. P. Petrov, Astron. Zh. (2010, in press).

2. P. Artymowicz and S. H. Lubow, Astrophys. J. 467, L77 (1994).

3. O. Yu. Barsunova, V. P. Grinin, and S. G. Sergeev, Astrofizika 48, 5 (2005).

4. M. R. Bate and I. A. Bonnell, Mon. Not. R. Astron. Soc. 285, 33 (1997).

5. C. J. Burrows, J. E. Krist, K. R. Stapelfeldt and WFPC2 InvestigationDefinition Team,Bull. Am. Astron. Soc. 27, 1329 (1995).

6. R. E. Cohen, W. Herbst, and E. L. Willams, Astrophys. J. 596, L243 (2003).

7. T. V. Demidova, Astrofizika 52, 623 (2009).

8. T. V. Demidova, N. Ya. Sotnikova, and V. P. Grinin, Pis'ma Astron. Zh. 36, No. 6, 445 (2010a) [Astron. Lett. 36, No. 422 (2010)].

9. T. V. Demidova, V. P. Grinin, and N. Ya. Sotnikova, Pis'ma Astron. Zh. 36, No. 7, 526 (2010b) [Astron. Lett. 36, No. 7, 498 (2010)].

10. V. P. Grinin, A. N. Rostopchina, and D. N. Shakhovskoi, Pis'ma Astron. Zh. 24, 925 (1998) [Astron. Lett. 24, 802 (1998)].

11. V. P. Grinin, E. Stempels, G. Gahm, et al., Astron. Astrophys. 489, 1233 (2008).

12. J. D. Larwood and J. C. P. Papaloizou, Mon. Not. R. Astron. Soc. 285, 288 (1997).

13. D. Mouillet, J. D. Larwood, J. C. B. Papaloizou, and A. M. Larange, Mon. Not. R. Astron. Soc. 292, 896 (1997).

14. A. Natta and B.Whitney, Astron. Astrophys. 364, 633 (2000).

15. S. Nordhagen, W. Herbst, E. C. Willams, and E. Semkov, Astrophys. J. 646, L151 (2006).

16. G. Pojmanski, Acta Astron. 52, 397 (2002).

17. A. N. Rostopchina, Astron. Zh. 76, 136 (1999) [Astron. Rep. 43, 113 (1999)].

18. A. N. Rostopchina, V. P. Grinin, D. N. Shakhovskoi, et al., Astron. Zh. 84, 60 (2007) [Astron. Rep. 51, 55 (2007)].

19. B. A. Smith and R. J. Terrile, Science 226, 1421 (1984).

20. N. Ya. Sotnikova, Astrofizika 39, 259 (1996).

21. N. Ya. Sotnikova and V. P. Grinin, Pis'ma Astron. Zh. 33, 667 (2007) [Astron. Lett. 33,594 (2007)].

Translated by N. Samus' 

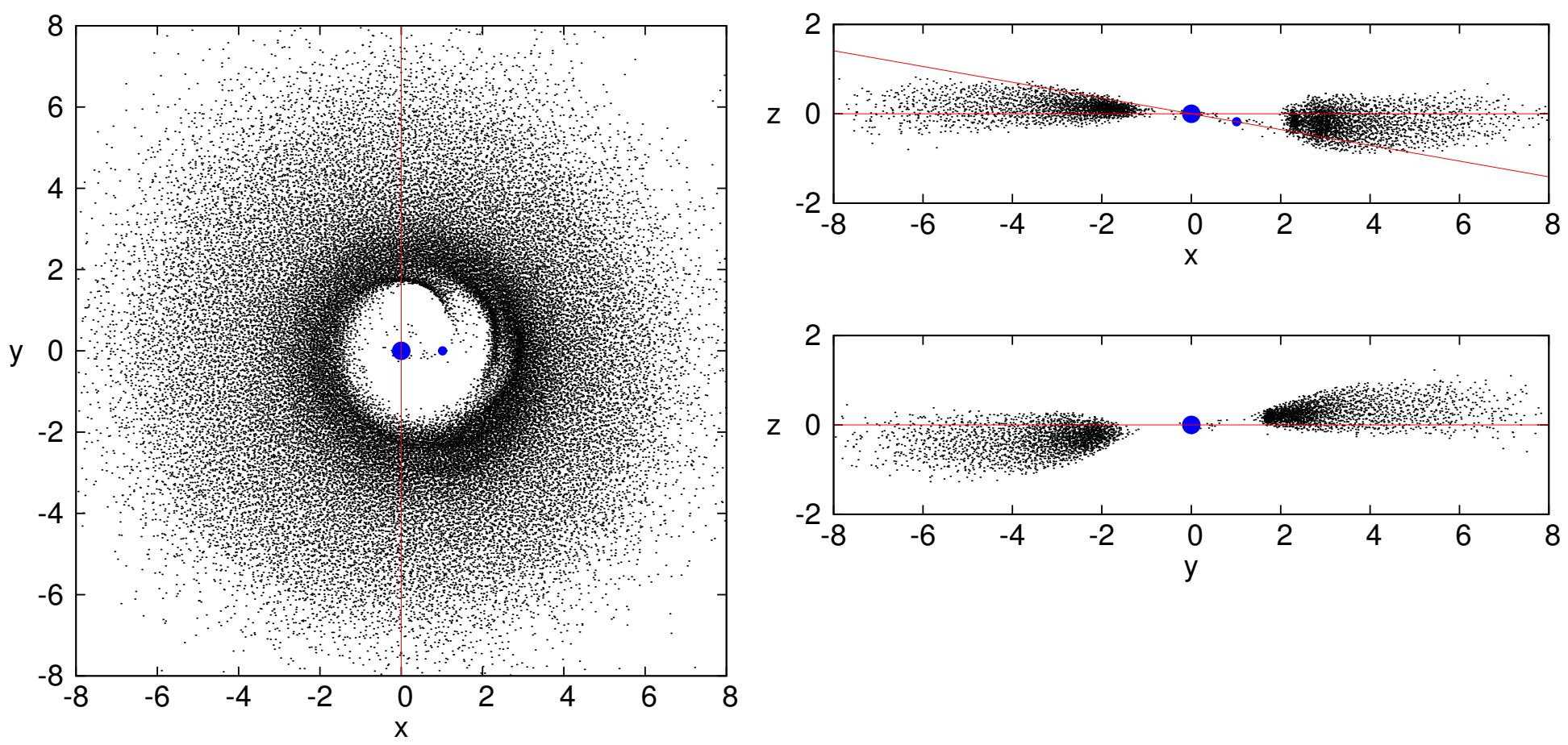

Figure 1: Distribution of matter in model $1(q=0.1)$ : Left - top view; right - disk sections in the XZ and YZ planes. The scale along the axes is given in units of the orbital semimajor axis. The line of nodes coincides with the $\mathrm{Y}$ axis. The binary rotates clockwise 

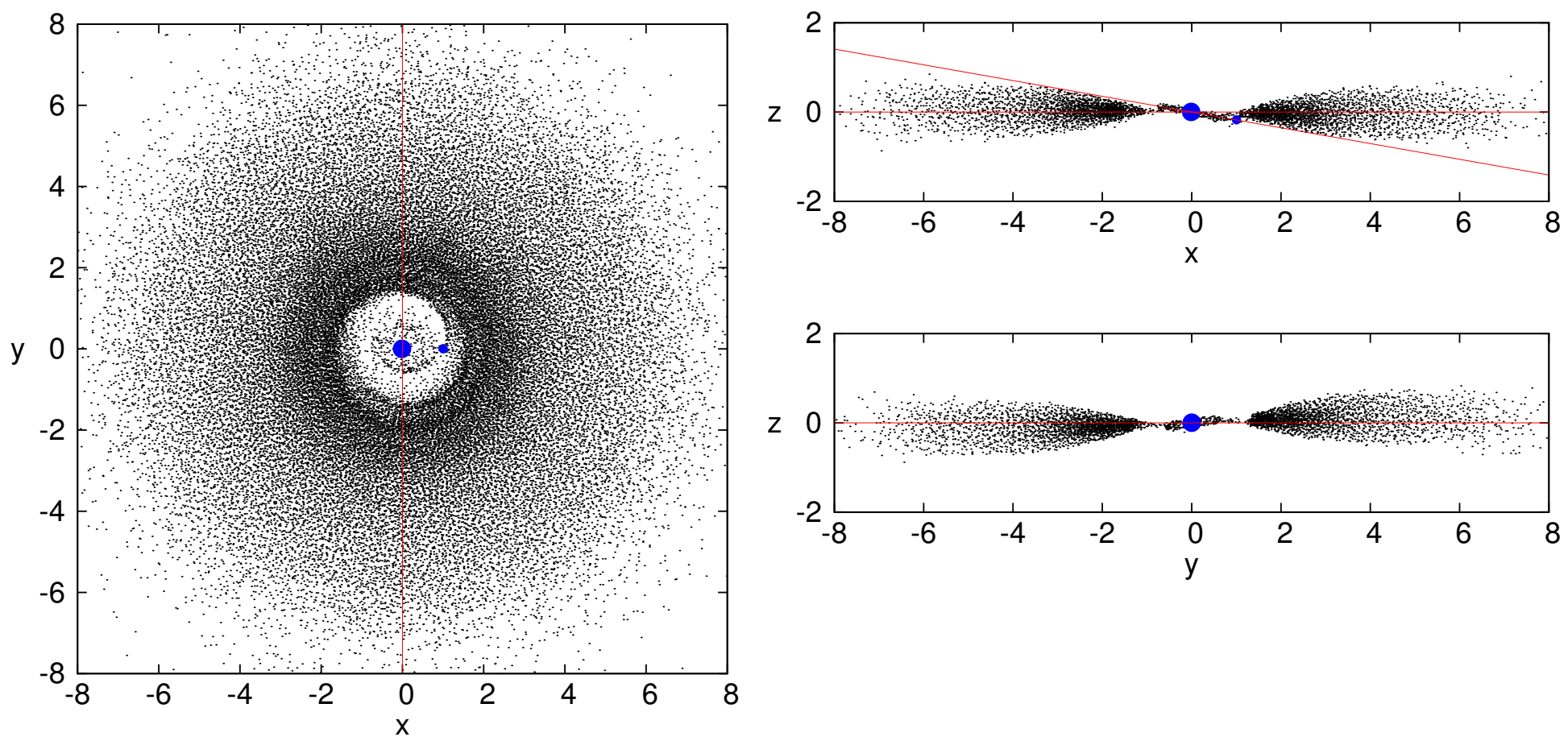

Figure 2: Same as Fig. 1 for model $2(q=0.01)$. 

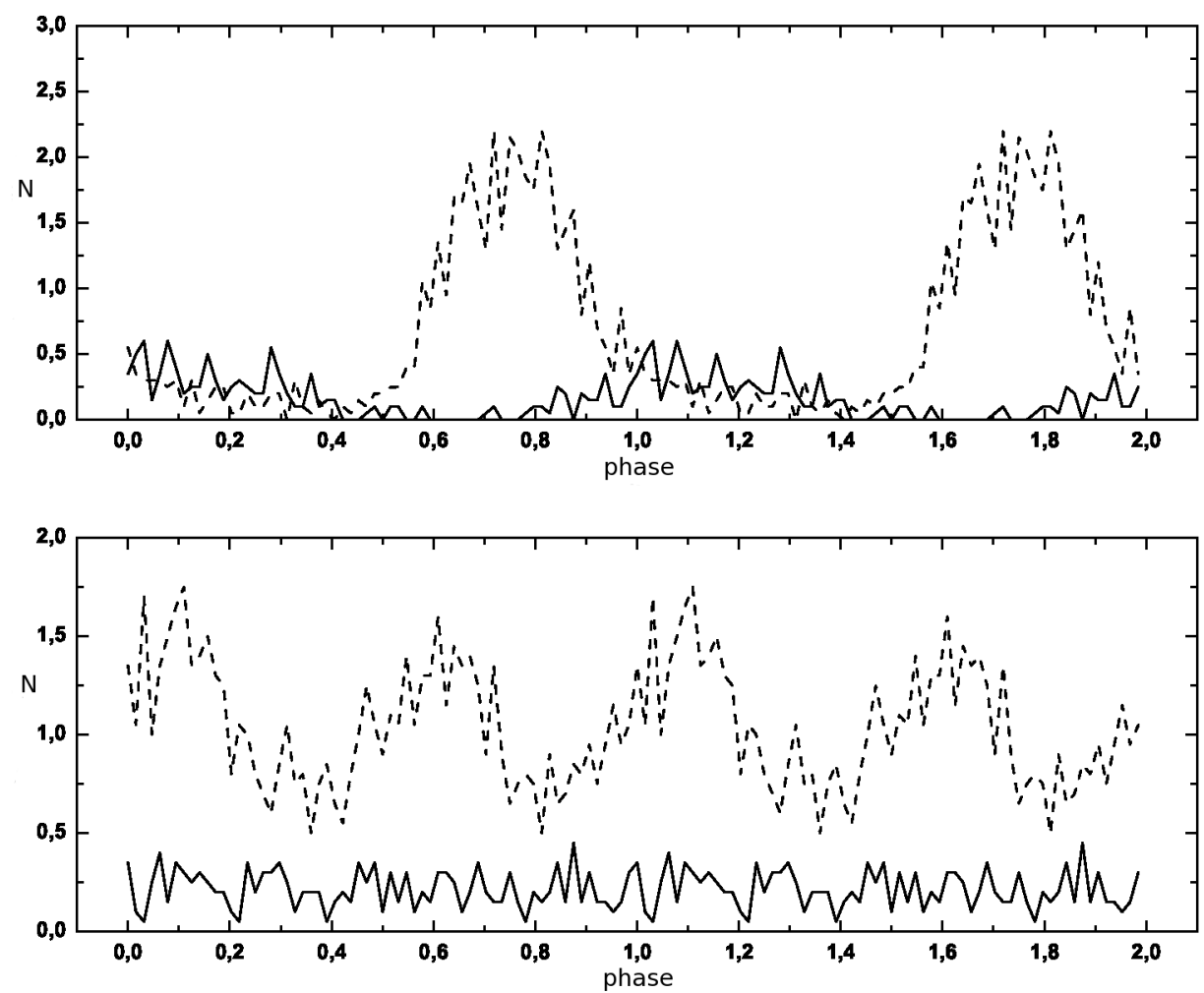

Figure 3: Phase dependences of the accretion rate onto the primary (solid lines) and secondary (dashed lines) components of the binary: top - model 1 and bottom - model 2. The scale along the vertical axis gives the number of test particles accreting onto the binary components in $1 / 64$ of the orbital period. 

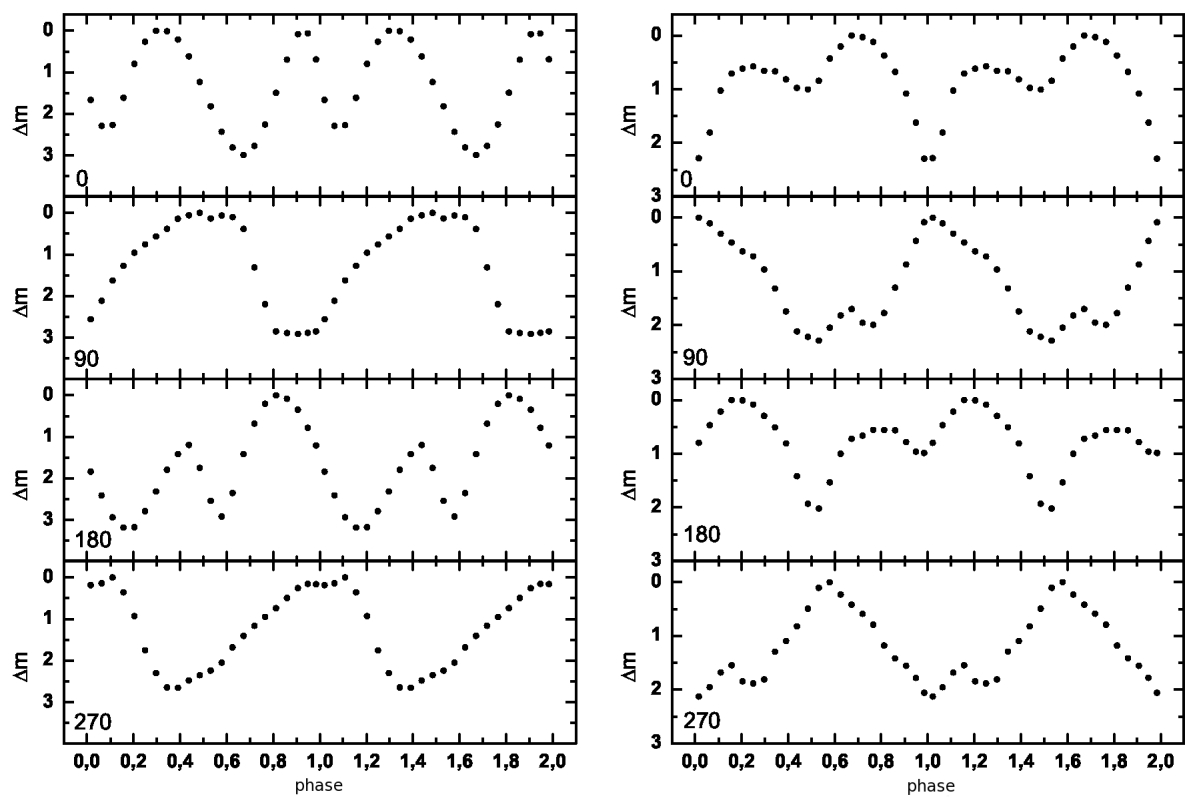

Figure 4: Left - light curves in model $1(q=0.1)$ for four orientations of the line of nodes $(\phi=0,90,180$ and $\left.270^{\circ}\right)$ at $i=0$; right - the same formodel $2(q=0.01)$. In both cases $\dot{M}_{a}=10^{-9} M_{\odot} / \mathrm{yr}$. 

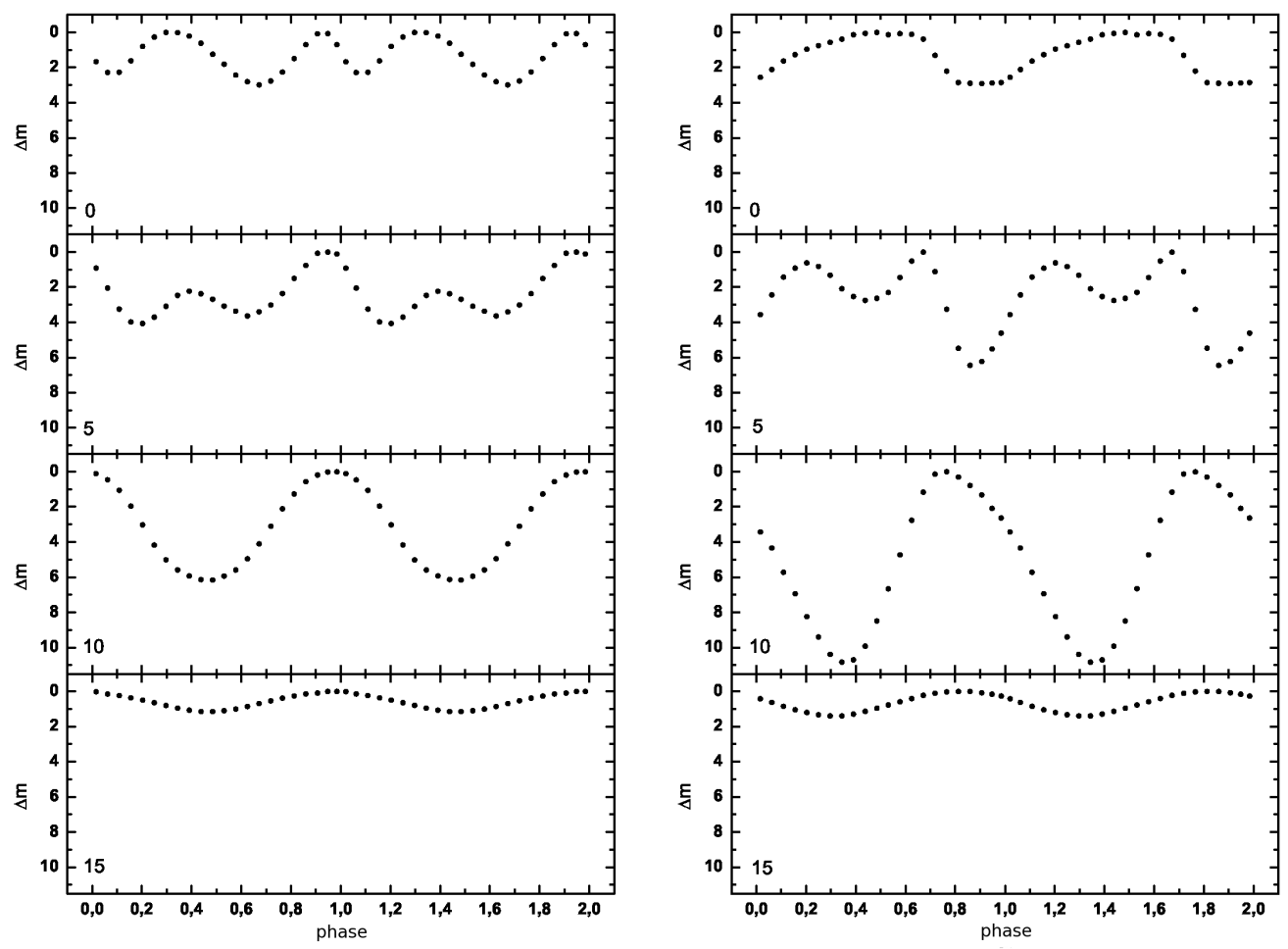

Figure 5: Light curves in model $1\left(q=0.1 ; \dot{M}_{a}=10^{-9} M_{\odot} / \mathrm{yr}\right)$ for various inclinations of the line of sight to the orbital plane and two positions of the line of nodes: left $-\phi=0$, right $-\phi=90^{\circ}$. 

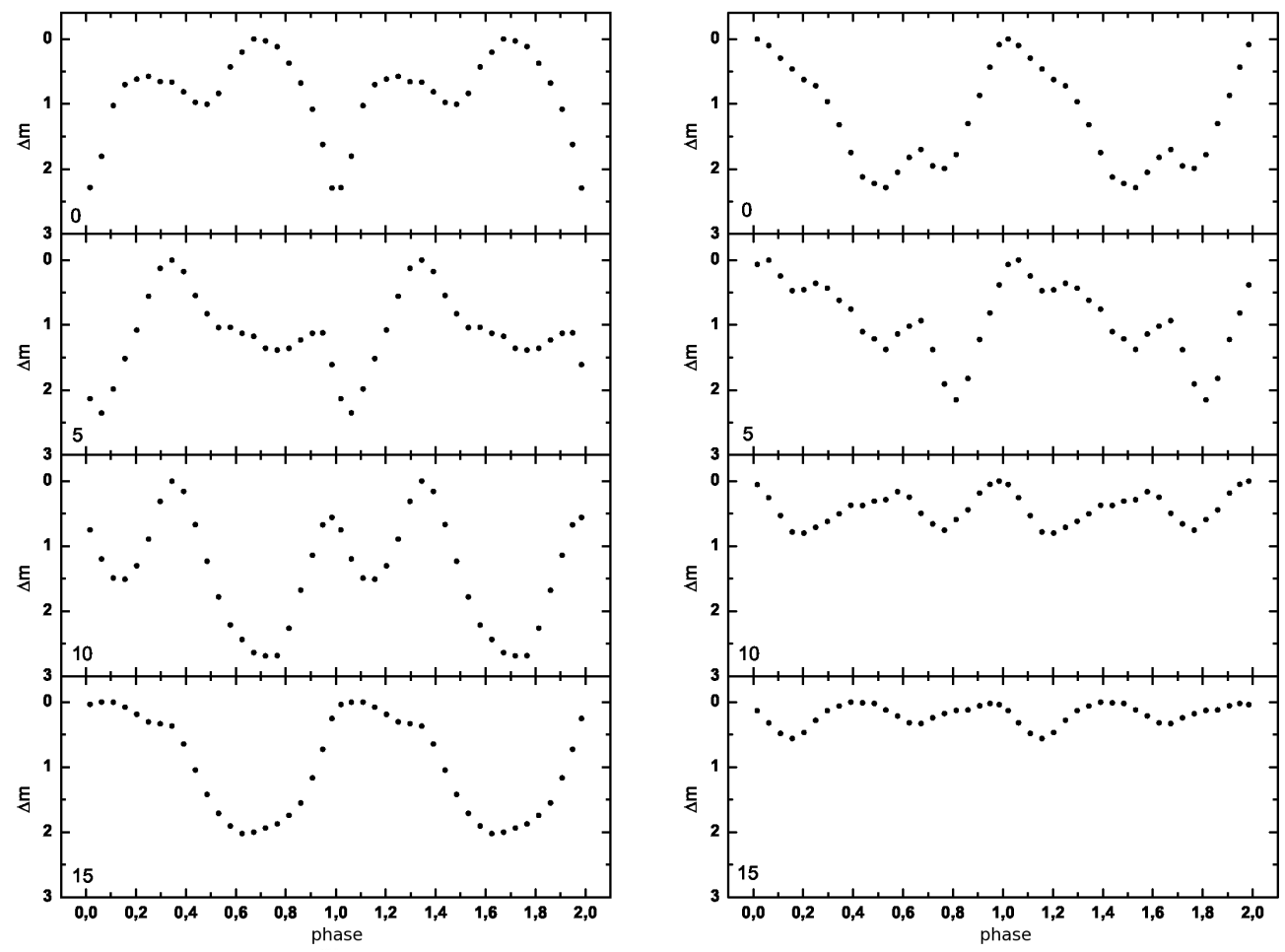

Figure 6: Light curves in model $2\left(q=0.01 ; \dot{M}_{a}=10^{-9} M_{\odot} / \mathrm{yr}\right)$ for various inclinations of the line of sight to the orbital plane and two positions of the line of nodes: left $-\phi=0$, right $-\phi=90^{\circ}$. 

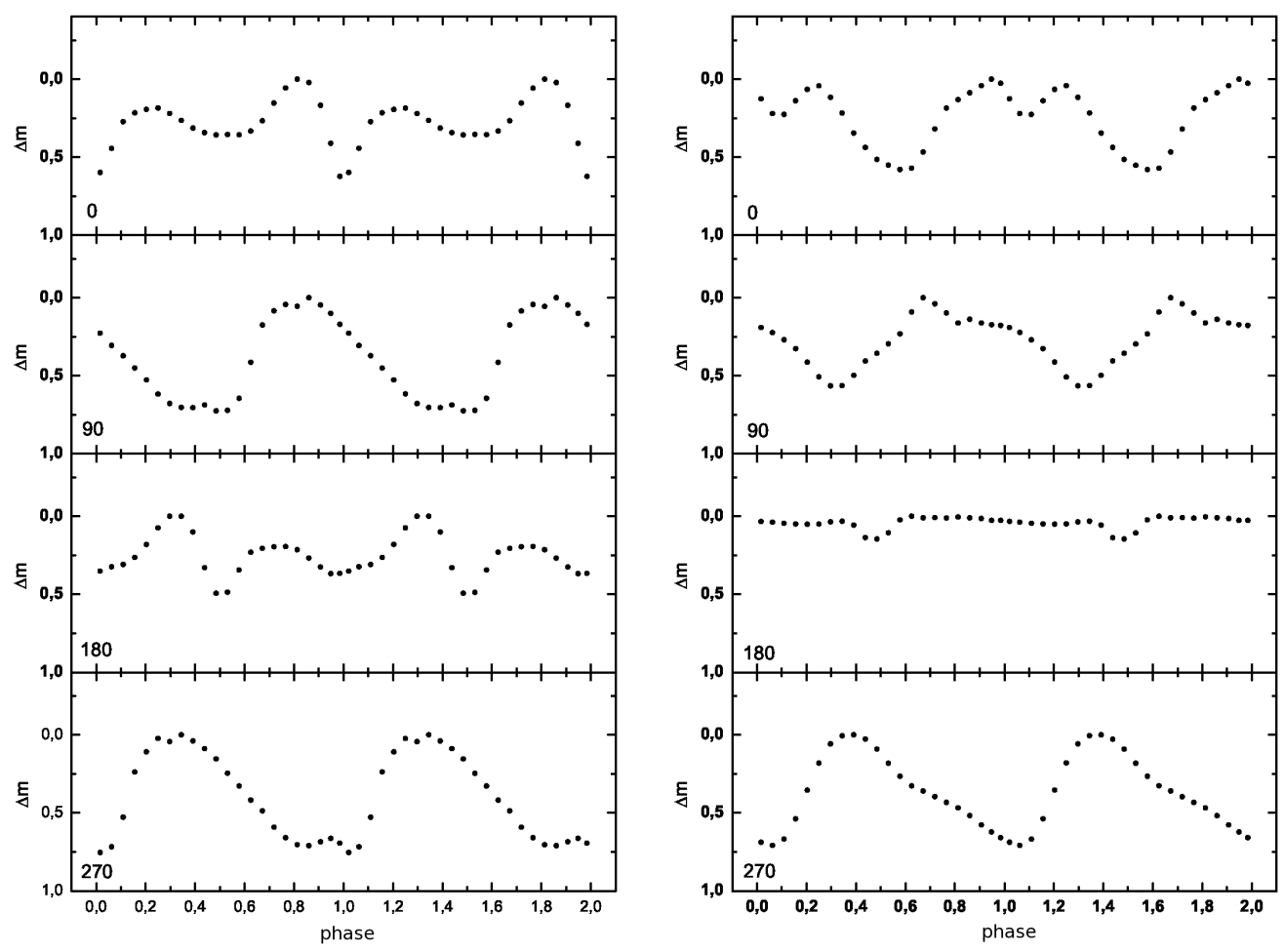

Figure 7: Light curves in model $3\left(q=0.003 ; \dot{M}_{a}=10^{-10} M_{\odot} /\right.$ yr $)$ for two inclinations of the orbital plane to the line of sight: left $-i=0$, right $-i=5^{\circ}$ and four positions of the line of nodes: $\phi=0,90,180 \& 270^{\circ}$. 